\title{
FloodRisk I und II: Grundlagen für ein integriertes Hochwassermanagement in Österreich
}

Zusammenfassung: Das Projekt „FloodRisk II - Vertiefung und Vernetzung zukunftsweisender Umsetzungsstrategien zum integrierten Hochwassermanagement" bestand aus 45 Teilprojekten, die in 8 Workpackages zusammengefasst wurden. Es liefert wesentliche Empfehlungen zu allen Bereichen des integrierten Hochwassermanagements. Neben den „klassischen“ Themen des Hochwassermanagements findet sich nun in FloodRisk II auch ein eigenes Arbeitspaket für „Ökologie“ und ein ausführlicher rechtlicher Teil. Abschließend beinhaltet FloodRisk II eine Umsetzungsstrategie inklusive einer Darstellung der bisher getätigten Maßnahmen als Folge von FloodRisk I (Habersack et al., 2004) und des Verbesserungsbedarfs sowie erweiterte und neue Empfehlungen.

FloodRisk I and II: Fundamentals for integrated flood management in Austria

Summary: The project "FloodRisk II - Intensification and integration of future oriented implementation strategies for the integrated flood management" consisted of 45 sub projects, which were summarized in 8 work packages. The project contains essential suggestions in all areas of the integrated flood management. Besides ecology a new theme in FloodRisk II is law. Finally FloodRisk II contains a detailed implementation strategy including a discussion of the so far realized measures as a consequence of FloodRisk I (Habersack et al., 2004) as well as the demand for further improvements and new suggestions in all aspects of integrated flood management.

\section{Einleitung, Problemstellung und Ziele}

Im Projekt „Analyse der Hochwasserereignisse vom August 2002 - FloodRisk I“ (Habersack et al, 2004) konnten wesentliche Erkenntnisse, Defizite und der daraus folgende Handlungsbedarf für ein zukunftsorientiertes, integriertes Hoch-

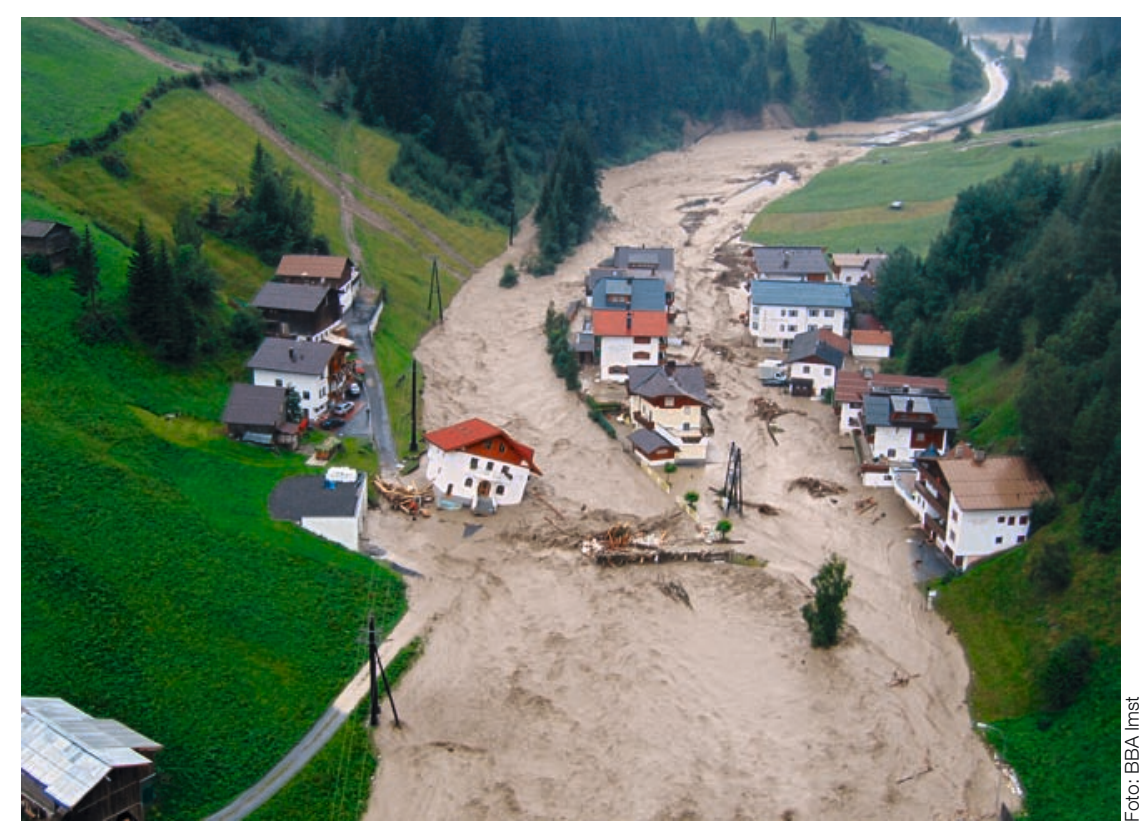

Abb. 1: Hochwasser 2005 in Nederle (Paznauntal), Trisanna, Tirol.

wassermanagement definiert werden.

Unmittelbar nach Abschluss des Projektes FloodRisk I trat im August 2005 ein weiteres extremes Hochwasserereignis, diesmal vorrangig im alpinen Bereich Westösterreichs, auf (Habersack et al, 2006, Abb. 1). Dabei kam es zu spezifischen Problemen, u.a. auf Grund des Feststofftransportes. 2006 trat an der March ein Extremhochwasser auf, wo viele Dammbrüche Ursache von Schäden waren und 2009 war der Osten und Südosten Österreich neuerlich von zahlreichen größeren Hochwasserereignissen betroffen. In der Folge von FloodRisk I stellte sich heraus, dass bestimmte Themenstellungen noch nicht ausreichend behandelt wurden, wie beispielsweise die Bereiche Klimawandel und Hochwasser, Ökologie und Hochwasser oder auch rechtliche Aspekte in Zusammenhang mit Hochwassermanagement. Weiters führt die Implementierung der EU-Hochwasserrichtlinie (Richtlinie 2007/60/EG) zu neuen Herausforderungen an das integrierte Hochwassermanagement. Folgende Ziele stellten sich im Rahmen von FloodRisk II:
- Schließen von Lücken aus FloodRisk I

- Bearbeitung neuer Themenbereiche (Klimawandel, Ökologie, Recht etc.)

- Ableitung von konkreten Umsetzungsvorschlägen

\section{Struktur von FloodRisk II}

In FloodRisk II wurden in acht Workpackages 45 Projekte von 60 Organisationen über alle institutionellen Grenzen hinweg von 140 Personen durchgeführt (Abb. 2).

\section{Ausgewählte Empfehlungen aus FloodRisk II}

\subsection{Meteorologie und Hydrologie}

- Die Variabilität der Klimaszenarien lässt aufgrund der unterschiedlichen Ergebnisse keine österreichweit einheitliche Aussage und darauf begründete Vorgangsweise hinsichtlich einer Veränderung der Bemessungsabflüsse zu. Ein Sicherheitszuschlag für Klimaänderung zu den Bemessungswerten erscheint aufgrund der vorhandenen Datenlage nicht gerechtfertigt. 
- Die in der Vergangenheit eminent große Variabilität der Hochwässer steht auch in Zukunft bei der Ermittlung von Bemessungswerten im Vordergrund und die berechneten Unterschiede der klimawandelrelevanten Wenn-Dann-Szenarien sind demgegenüber klein. Bei der Bestimmung von Bemessungswerten des Hochwassers sind das Auftreten von Hochwasserdekaden jedenfalls zu berücksichtigen, die Verwendung einer möglichst weitreichenden Datenbasis anzustreben und hochwasserrelevante, regional-spezifische Informationen zu verwenden. Es ist zu erwarten, dass damit die Unsicherheit in den Bemessungswerten stärker reduziert werden kann als durch Berücksichtigung unsicherer, klimainduzierter Trends.

- Hinsichtlich der Berücksichtigung von Klimaszenarien ist weiters zu beachten, dass die Auswirkungen der Raumnutzung wesentlich stärkere Folgen für das Hochwasserschadenspotenzial haben werden als ein unsicheres Klimasignal.

- Nicht nur in Wildbacheinzugsgebieten sondern auch generell ist danach zu trachten, eine Verschärfung der kritischen Abflusssituation durch weitere Versiegelungseffekte zu verhindern und den Rückhalt im Einzugsgebiet der Gewässer optimal zu nutzen.

- Die Veränderung des Waldes in Richtung dichter, kleinflächig und stufig strukturierter Wald wirkt sich hydrologisch günstig aus und kann zur Dämpfung kleinerer und mittlerer Hochwasserwellen führen.

\subsection{Geomorphologie}

- Freihaltung des minimalen flussmorphologischen Raumbedarfs: Erhaltung/Herstellung eines minimalen Sicherheitsstreifens der mindestens der 1 - bis 3-fachen Flussbreite (aktuelle Breite zwischen Böschungsoberkanten) links- und rechtsufrig entspricht und mit absolutem Bebauungsverbot belegt ist. Insgesamt sollte minimal die 3- bis 7 -fache Flussbreite freigehalten werden, um Flächen für die morphologischen Veränderungen bei großen Hochwasserereignissen zur Verfügung $\mathrm{zu}$ haben und damit Schäden zu verringern. Nach Möglichkeit soll der maximale flussmorphologische Raumbedarf im Freiland angestrebt werden.

- Die Erhaltung oder Wiederherstellung des Sedimentkontinuums und der gewässertypischen Morphodynamik ist anzustreben.

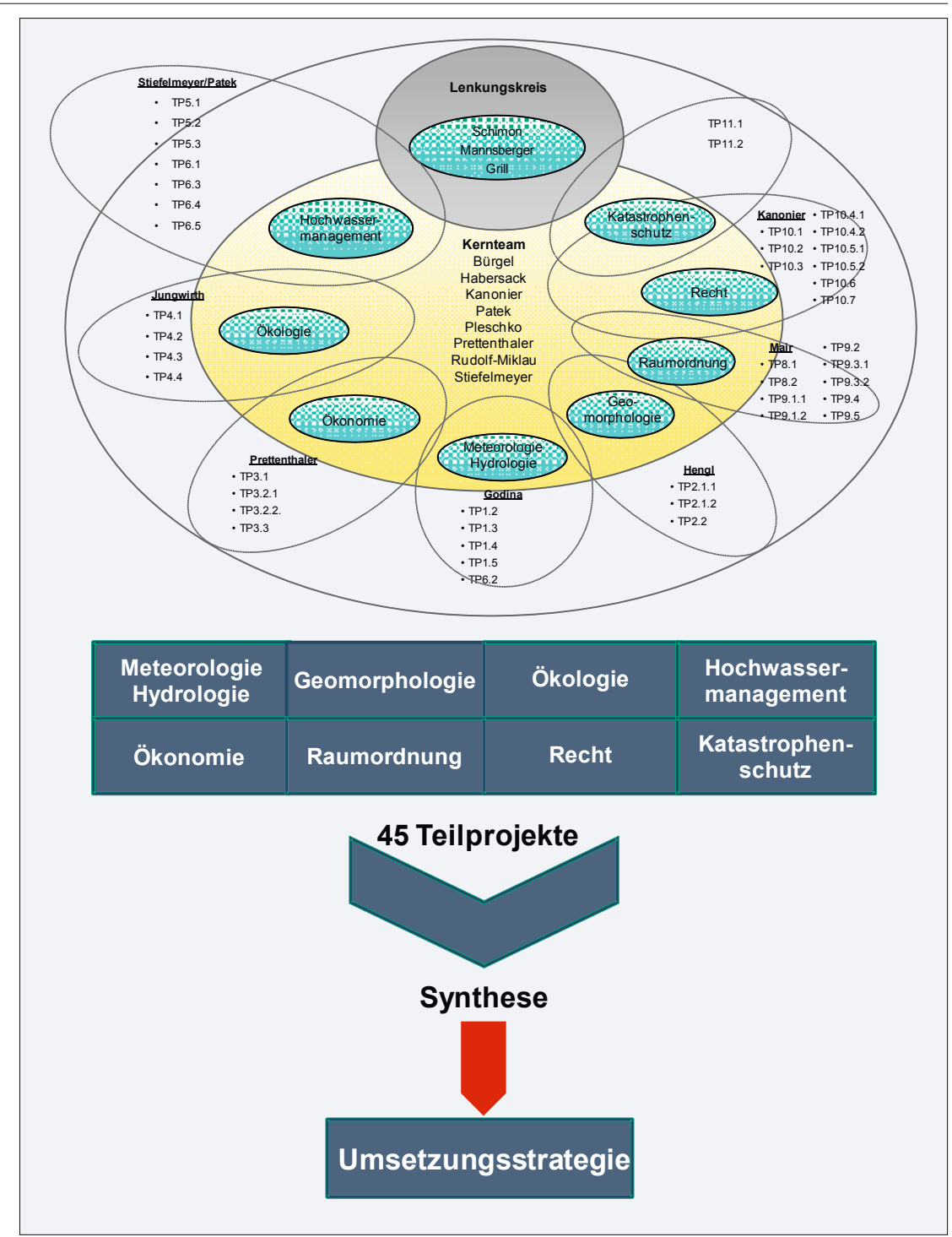

Abb. 2: FloodRisk II: Struktur, Workpackages und Teilprojekte.

- Bestehende Einbauten (Rechen, Netze, Sperren etc.) und hydraulische Engstellen (Brücken, Durchlässe usw.) sind betreffend Verklausungsrisiko regelmäßig zu kontrollieren. Bei Bedarf ist der Rückhalt des Wildholzes durch geeignete waldbauliche, ingenieurbiologische und technische Maßnahmen sicherzustellen.

\section{3. Ökologie}

- Eine praktische Umsetzung eines räumlich differenzierten Vegetationsmanagements, welches je nach Gefährdungssituation Strecken mit unterschiedlichen Managementempfehlungen definiert, ist anzustreben.

- Der Schutz und die Erhaltung der vorhandenen gewässertypspezifischen Flussabschnitte, die Erhaltung und
Vergrößerung des Fluss-Auenraumes entsprechend der lateralen Ausdehnung und der Morphodynamik des Flusstyps sind zu forcieren.

- Restaurationsmaßnahmen sind an degradierten Flussabschnitten unter Betrachtung des gesamten potenziellen Fluss-Auensystems durchzuführen. Der morphologische Flusstyp ist zu initiieren bzw. entwickeln und das potenzielle Fluss-Auensystems ist zu restaurieren inklusive der Vergrößerung bzw. Wiederanbindung von Überflutungsräumen bzw. Initiierung oder Anlage von auentypischen Habitaten.

- Die noch bestehenden Auen in Österreich, welche zumeist mehrere FFH (Fauna-Flora-Habitat) Lebensraumtypen beheimaten, sind zu schützen. Überflutungsflächen sind aus Sicht des Hochwasserschutzes und zur Verbesse- 
rung der Hydrologie für die Auen - insbesondere dort wo sie außerhalb der bestehenden Hochwasserschutzdämme liegen - wieder zu gewinnen. Der Eintrag von Nährstoffen in Auensysteme ist zu kontrollieren und zu minimieren.

- Eine Extensivierung der Umlandnutzung in der $\mathrm{HQ}_{30}$-Zone ist anzustreben.

\subsection{Hochwassermanagement}

- Raumplanerische, nicht-bauliche Maßnahmen sollten technischen Maßnahmen vorgezogen werden. Die wirksamste Art das Schadenspotenzial niedrig zu halten besteht in der Freihaltung von Überflutungsflächen.

- Mobiler Hochwasserschutz sowie Objektschutz stellen für den bereits bebauten Bereich eine sinnvolle Ergänzung nichtstruktureller und struktureller Hochwasserschutzmaßnahmen zur weiteren Risikoreduktion dar.

- Da Schutzbauwerke, wie alle technischen Bauwerke, eine beschränkte Lebensdauer besitzen und sich im Laufe der Zeit abnutzen, ist die Funktionserfüllung vom Zustand des Bauwerkes abhängig. Um diesen auf einem akzeptablen Niveau zu halten, müssen die Bauwerke regelmäßig überwacht und instand gesetzt werden. Die normative Regelung der Bauwerkserhaltung (z. B. ONR 24803) stellt dabei eine wichtige Grundlage für die Erhaltungsverpflichteten dar.

- Eine systematische Zustandserhebung von bestehenden Hochwasserschutzdämmen ist als Basis für die Bewertung des Ist-Zustandes unumgänglich. Ebenso sind Vorkehrungen gegen unkontrolliertes Überströmen von Hochwasserschutzdämmen zu treffen.

- Bereits vor Hochwasserereignissen sollten Planungen für mögliche Sofortmaßnahmen a priori zur Verfügung stehen, um im Anlassfall auf bestehenden Projekten aufzubauen und damit den Zeitraum der Umsetzung wesentlich zu verringern.

\section{5. Ökonomie}

- Aus volkswirtschaftlicher Sicht gilt es vor allem, die vorhandenen Ressourcen bestmöglich einzusetzen. Die Bereitstellung einer soliden Informationsbasis über die Gefährdung vor Ort ist die dringlichste Aufgabe. Mit der nahezu flächendeckend vorhandenen Gefahrenzonenplanung ist eine hervorragende Grundlage für das Risikomanage- ment geschaffen worden. Dieses Instrument aber muss weiter entwickelt werden und die Pläne müssen laufend den neuen Erkenntnissen und Gegebenheiten angepasst werden.

- Die wirtschaftliche Entwicklung nimmt nicht in vielen Regionen zu. In der vorausschauenden Planung muss diesem Umstand Rechnung getragen werden. Die höchste Priorität der Maßnahmensetzung sollten daher jene Gebiete haben, in denen pro investiertem Euro die meisten Schäden vermieden werden können. Das dazu eingesetzte Verfahren ist die Kosten-Nutzen-Analyse. Die Anwendung dieser Methode muss ebenso den laufenden Änderungen Rechnung tragen und kontinuierlich verbessert werden.

- Die Erfahrungen aus den durchgeführten Dokumentationen der Hochwässer 2002 und 2005 zeigen, dass für eine effiziente Vorgangsweise eine standardisierte Dokumentation von Hochwasserereignissen erforderlich ist. Aufgrund der heterogenen Betrachtungsweisen und methodischen Zugänge entstanden Dokumentationen mit unterschiedlichen Ergebnissen. Diese machen sich sowohl in der Detailschärfe der Bearbeitung als auch durch unterschiedliche Schwerpunkte bemerkbar. Der erarbeitete Vorschlag einer österreichweit einheitlichen Vorgangsweise für die Bundeswasserbauverwaltung ist anzustreben.

\subsection{Raumordnung}

- Ein eindeutiges Bekenntnis der politischen Verantwortlichen auf allen Ebenen (kommunal, regional, landesweit, national) - insbesondere auch in der Vollziehung - zur Flächensicherung und zu hochwasserverträglichen Nutzungen im Flussraum und nachhaltigen Hochwasserschutz ist für die Umsetzung eines wirkungsvollen, integrierten, nachhaltigen Hochwasserrisikomanagements entscheidend.

- Zwischen den verschiedenen Fachbereichen besteht in Österreich - nach wie vor - ein beachtlicher Abstimmungs- und Harmonisierungsbedarf. Eine verstärkte Kooperation insbesondere von Raumplanung und (Schutz-) Wasserwirtschaft im Rahmen integrativer, einzugsgebietsbezogener Planungsinstrumente ist anzustreben. Um den Anforderungen einer zeitgemäßen Planung gerecht zu werden, gilt es, die vorhandenen Planungsins- trumente $\mathrm{zu}$ adaptieren bzw. neue Planungsinstrumente zu entwickeln und vor allem in der praktischen Umsetzung fachübergreifend abgestimmte Lösungsansätze zu forcieren.

- Die raumordnungsgesetzlichen Ziele sollten verstärkt die Freihaltung von Überflutungsflächen priorisieren und Aussagen für den Umgang mit gefährdetem Widmungs- und Baubestand enthalten, um die öffentlichen Interessen an einer Reduktion unbebauter Bauflächen einerseits sowie die Sicherstellung gefährdeter Siedlungsbereiche andererseits zu verdeutlichen.

- Interkommunale Kooperationen sind zu fördern, zumal vielfach nur das $\mathrm{Zu}-$ sammenwirken mehrerer Gemeinden wirkungsvolle Maßnahmen ermöglicht. Förderungen für Kooperationen oder eine Verknüpfung mit Finanzierungsmöglichkeiten können wesentliche Anstöße für eine interkommunale Kooperation in der Hochwasserflächenvorsorge sein. Ausgleichsmaßnahmen im Sinne von finanziellen Transfers zwischen Gemeinden sind umzusetzen bzw. zu entwickeln.

- Neben der Freihaltung von Retentionsund Überflutungsflächen sind verstärkt Lösungsstrategien für durch Hochwasser gefährdete Siedlungen und Bauten zu entwickeln, wobei auch Absiedelungen - die rechtlichen Grundlagen dafür sind noch zu schaffen - als Alternative zu (technischen) Hochwasserschutzmaßnahmen vermehrt in Betracht zu ziehen sind.

- Die gesetzlichen Grundlagen für die Freihaltung von bedeutsamen Überflutungsflächen in der überörtlichen Raumplanung sind zu schaffen bzw. zu verbessern. Der planerische Fokus ist verstärkt auf Talräume mit starker Raumentwicklung zu legen, wobei besonderes Augenmerk auf einzugsgebietsbezogene und über die Gemeindegrenzen hinweggehende Betrachtungen gelegt werden soll.

\subsection{Recht}

- Die Hochwasserrichtlinie (HWRL) mahnt eine stärkere staatliche Verantwortung für das Hochwasserrisikomanagement ein, als derzeit im Wasserrechtsgesetz (WRG) vorgesehen, und Entscheidungen des Europäischen Gerichtshofs für Menschenrechte deuten ebenfalls in diese Richtung. Die Aufgaben des Staates sollten sich dabei insbesondere auf Informations- und Warn- 
pflichten sowie auf die mittelfristige übergeordnete Planung beziehen, wie sie die HWRL bei Hochwasserrisikomanagementplänen ohnehin vorsieht. Eine staatliche Verpflichtung zur Ausführung und Finanzierung von Schutzmaßnahmen ist im WRG nicht anzustreben.

- Das derzeitige WRG ist stark an der Vorstellung des Hochwasserschutzes durch bauliche Maßnahmen orientiert. Die Freihaltung von Überflutungsflächen findet im WRG zu wenig Berücksichtigung. Daher sollte der Grundsatz des Vorranges nicht baulicher Maßnahmen in Form einer Zielbestimmung ins WRG aufgenommen werden.

- Das WRG sollte der Schaffung und Erhaltung von „Überflutungsflächen“ stärkere Bedeutung zumessen und diese als eigenständige Schutzmaßnahme in das WRG aufnehmen (Einbeziehung der Summationseffekte).

- Die Ausweisung der Hochwassergefahr (z.B. durch Gefahrenzonenpläne, Gefahrenkarten, Risikokarten) muss in Hinkunft normativ aufgewertet werden. Allerdings bedarf es dazu klarer (einfach-)gesetzlicher Vorgaben. Es empfiehlt sich, das gestufte Verfahren nach der HWRL im WRG nachzuzeichnen.

- Gemeinden haben, auch aus Haftungsgründen, unbedingt dafür Sorge zu tragen, dass potenzielle Gefährdungsbereiche allgemein für die Bevölkerung ersichtlich werden und die Bereitstellung von Informationen über Naturgefahren im Sinne der öffentlichen Informationspflicht so umfassend wie möglich erfolgt.

- Die Raumordnungsgesetze der Länder sollten ihre Regelungen für den Umgang mit Bauland in Gefährdungsbereichen präzisieren und insbesondere Änderungs- und Rückwidmungsbestimmungen eindeutiger festlegen.

- Soweit Bauführungen in Überflutungsflächen rechtlich zulässig sind, sollte verpflichtend eine hochwassersichere Bauweise angeordnet werden. In allen Bauordnungen sollen die Baubehörden ermächtigt werden, erforderlichenfalls nachträgliche (Schutz-) Maßnahmen in Form von Auflagen/ Bedingungen vorzuschreiben.

\subsection{Katastrophenmanagement}

- Erarbeitung einer längerfristigen Strategie durch den weiteren Ausbau des staatlichen Krisen- und Katastrophenschutzmanagements unter Einbindung anderer Bundesministerien, der Bun- desländer und der Dachorganisationen der großen Einsatzorganisationen.

- Effizienzsteigerung im staatlichen Katastrophenschutzmanagement durch die Schaffung eines gemeinsamen Informationssystems zur Unterstützung von Entscheidungsprozessen, das relevante Informationen aus den Bereichen Meteorologie, Hydrologie, Verkehr, Einsatzführung etc. bündelt und auf das Katastrophenschutzbehörden auf verschiedenen Verwaltungsebenen zugreifen können.

- Ein zentraler Aspekt - insbesondere für das Management von Naturkatastrophen - ist die flächendeckende Versorgung mit Einrichtungen des Katastrophenschutzes, die nur mit ehrenamtlichen Strukturen möglich ist. Der Aufrechterhaltung des ehrenamtlichen Engagements im Katastrophenschutz kommt daher längerfristig eine wesentliche Bedeutung für den Erhalt der Qualität des gesamten Systems zu.

- Bei der Organisation im Hochwasserkatastrophenmanagement gibt es bereits zahlreiche Strategiepapiere, in die auch die Erfahrungen aus dem Hochwasserereignis 2002 eingeflossen sind. EDV-unterstützte Programme (Katastrophenschutzmanagementsysteme) können gerade auch auf Gemeindeebene ihren Beitrag dazu leisten, um die überaus komplexen Abläufe des Katastrophenschutzmanagements überschaubar betreiben zu können. Diese Erfahrungen und Ergebnisse, die in Pilotgemeinden bereits Anwendung finden, sollen den Gemeinden noch intensiver angeboten und näher gebracht werden (Alarm- und Warnpläne).

- Die Lamellenprognose bzw. Lamellenberechnung kann ein wesentliches Planungsinstrument für die Gefahrenanalyse und die Maßnahmenplanung im Katastrophenschutzmanagement auf lokaler Ebene sein.

\section{Umsetzungsstrategie}

\subsection{Umsetzungsstrategie aus FloodRisk I und II}

Grundsätzlich gilt, dass ein „absoluter“ (100\%-iger) Hochwasserschutz nicht möglich ist, vielmehr ist ein öffentliches Bewusstsein für das Restrisiko zu entwickeln.

Bereits in FloodRisk I wurden vielfältige Schritte vorgeschlagen, um vom sektoralen Hochwasserschutz zum integrierten Hochwassermanagement zu gelangen.
Dabei sind der Schutz von Menschenleben, der Schutz der Lebensgrundlagen und der Schutz der Gewässer sowie eine Minderung der Sachschäden, die Sicherung des Wiederaufbaus und Neuanfangs sowie die Nachhaltigkeit der Maßnahmen vorrangig.

\subsection{Integriertes}

Hochwassermanagement

Erfolgreiche Umsetzungsstrategien orientieren sich an einem integrierten Hochwassermanagement, welches sich auf Flussgebiete bezieht (Habersack et al., 2004). Integriertes Hochwassermanagement umfasst alle aktiven und passiven Schutzmaßnahmen mit permanenter und temporärer Wirkung und geht von der Idealvorstellung einer optimalen Kombination dieser Maßnahmen aus, um das Hochwasserrisiko so weit wie möglich bzw. auf ein akzeptables Ausmaß zu senken. Integriertes Hochwassermanagement bedeutet vor allem, das gemeinsame Ziel - eine möglichst große Sicherheit vor Hochwasser - durch ein sinnvolles Zusammenwirken von raumplanerischen, bautechnischen und organisatorischen Maßnahmen zu erreichen. Schutzwasserbauliche Maßnahmen sind ein wesentliches Standbein dieser Strategie, stellen aber keinesfalls ein Allheilmittel dar.

Integriertes Hochwassermanagement und daraus abgeleitete Maßnahmen setzen dabei klare, erkennbare Ziele voraus. Auf Basis der Ergebnisse aus FloodRisk I wurde vom Lebensministerium eine Strategie für ein integriertes Hochwassermanagement definiert.

Die Grundsätze für die Planung und Projektierung von Hochwasserschutzmaßnahmen sind im Bereich der BWV in der RIWA-T (Lebensministerium, 2006) und im Bereich der WLV in der Technischen Richtlinie für die Wildbach- und Lawinenverbauung (Fassung 2006) festgelegt, wo bereits wesentliche Erkenntnisse aus FloodRisk I Eingang fanden.

\subsection{Umsetzung des integrierten Hochwassermanagements seit FloodRisk I}

\subsubsection{Meteorologie und Hydrologie}

Im Bereich der Meteorologie wurden wesentliche Fortschritte erzielt bzw. Projekte betreffend Erstellung/Erneuerung von Prognosemodellen initiiert, abgeschlossen oder sind in Bearbeitung. An fast allen österreichischen mittleren und größeren Flüssen wurden Hochwasserprognosemo- 
delle eingerichtet oder befinden sich in Entwicklung. Auch im Bereich der Prognose und Frühwarnung von Sturzfluten und Hochwasserereignissen in Wildbacheinzugsgebieten konnten Fortschritte erzielt werden.

\subsubsection{Geomorphologie}

Im Bereich der Fließgewässermorphologie sind in der Praxis erste Veränderungen vorgenommen worden. Die Schaffung eines Sedimentkontinuums im Sinne der wasserwirtschaftlichen und ökologischen Bedürfnisse wurde dabei noch nicht eingehend diskutiert, steht aber in Verbindung mit dem Feststoffhaushalt und der Flussmorphologie (wichtig auch für NGP).

\subsection{3. Ökonomische Aspekte}

Diskussionen über den Aufbau einer bundeseinheitlichen Vorgehensweise bei der Erhebung und Analyse von Ereignis- und Schadensdaten sowie der Umgestaltung des Risikotransfermechanismus wurden begonnen. Derzeit liegen aber noch keine einheitlichen Vorgangsweisen vor, wie auch die Hochwässer 2005, 2006 und 2009 zeigten.

\subsubsection{Raumordnung}

In der Raumordnung gab es - je nach Bundesland unterschiedlich - deutliche Verbesserungen in den jeweiligen Raumordnungsgesetzen. Einzelne Bundesländer haben in ihren jüngsten Novellen ihre raumordnungsrechtlichen Zielkataloge überarbeitet und dem planerischen Umgang mit Naturgefahren höhere Bedeutung beigemessen. Teilweise wurden auch die Regelungen für Gefährdungsbereiche geändert.

\subsubsection{Hochwasserschutzmaßnahmen}

Österreichweit wird intensiv an der Ausweisung von Hochwasseranschlagslinien oder Gefahrenzonenplänen gearbeitet. Dabei erfolgt im Bereich der BWV länderweise eine unterschiedliche Vorgangsweise (Anschlagslinie oder GZP) bzw. Schwerpunktsetzung, was die flächendeckende Verfügbarkeit dieser Information betrifft. Im Kompetenzbereich der Wildbach- und Lawinenverbauung werden alle Gefahrenzonenpläne nach 2010 vorliegen.

Das Lebensministerium und der Verband der Versicherungsunternehmen
Österreich (VVO) starteten bereits im Spätherbst 2002 das Projekt „Hochwasserrisikozonierung Austria - HORA“, ein bundesweites Risikozonierungssystem für Naturkatastrophen mit dem besonderen Schwerpunkt Hochwasser.

Unter Einschluss der Themen Restrisiko/erhöhtes Risiko und erweiterte Planungsinstrumente ist 2006 eine neue technische Richtlinie im Bereich der BWV (RIWA-T) herausgegeben worden. Generell ist die RIWA-T als wesentliches Instrument zur Umsetzung von Empfehlungen aus FloodRisk anzusehen. Ebenso wesentlich sind die technischen Richtlinien der WLV.

Im Jahr 2005 erfolgte von Bundesseite eine Mittelaufstockung für Hochwasserschutzmaßnahmen von vormals etwa 50 Mio. $€$ auf nunmehr knapp 80 Mio. $€$ pro Jahr. Damit sollen bis 2016 die mittelfristigen Hochwasserschutzplanungen umgesetzt werden.

Im Bereich der Bundeswasserstraßenverwaltung (BMVIT) erfolgte die Ausarbeitung einer Bund-Länder-Vereinbarung, wodurch in den nächsten Jahren insgesamt Mittel in der Höhe von 450 Mio. $€$ für Hochwasserschutzmaßnahmen entlang der Donau sichergestellt sind.

\subsubsection{Katastrophenschutz}

Umfangreiche Verbesserungen fanden zum Katastrophenschutz statt. Beispielsweise wurden in NÖ und OÖ völlig neue Konzepte entwickelt, die derzeit schon umgesetzt werden oder sich in Erprobung befinden. Die in den ausgewählten Empfehlungen von FloodRisk I genannten länderübergreifenden Synergien sind allerdings nur vereinzelt festzustellen.

\subsection{Empfohlene Maßnahmen zur Weiterentwicklung der Strategien des integrierten Hochwassermanagements}

Die strategische Ausrichtung des Hochwassermanagements in Österreich beruht auf jahrzehntelanger Erfahrung, greift aber auch Erkenntnisse auf, die aus der Bewältigung der jüngsten Hochwasserkatastrophen gewonnen werden konnten. Die Zukunftsaufgaben liegen vor allem in einem stärkeren integrierten Hochwassermanagement unter Beteiligung der Bürgerinnen und Bürger aber auch in der fachlichen und inhaltlichen Weiterentwicklung.

Die 10 Strategien des Hochwasserschutzes für Österreich, die vom Bundesminister für Land- und Forstwirtschaft,
Umwelt und Wasserwirtschaft bereits 2004 vorgegeben wurden, werden in den nachfolgenden Kapiteln auszugsweise um weiterführende Umsetzungsempfehlungen aus FloodRisk II ergänzt.

\subsubsection{Die Grenzen des Schutzes und der Verantwortung der Beteiligten aufzeigen}

Aus FloodRisk II aber auch der Umsetzung der EU-Hochwasserrichtlinie (Richtlinie 2007/60/EG) ergeben sich Notwendigkeiten hinsichtlich der Untersuchung von seltenen Hochwasserereignissen (z.B. $\mathrm{HQ}_{300}$ ), der Erstellung von Gefahren- und Risikokarten und Hochwasserrisikomanagementplänen. Bei der Planung von Hochwasserschutzmaßnahmen soll zukünftig auch das Restrisiko, entweder im Überlastfall (Ereignis größer als Bemessungsereignis) oder bei Versagen der Anlage Berücksichtigung finden und den $\mathrm{Be}$ troffenen (der geschützten Bevölkerung) kommuniziert werden.

\subsubsection{Gefahrenkenntnis und Gefahrenbewusstsein fördern}

Die EU Hochwasserrichtlinie verstärkt die Umsetzung von Gefahrenkarten etc., so dass die Gefahrenkenntnis und das Gefahrenbewusstsein gefördert werden. Dazu gibt es in FloodRisk II konkrete Vorschläge zur Umsetzung einschließlich des rechtlichen Anpassungsbedarfs. Hinsichtlich Zusammenarbeit der Katastrophenschutzeinrichtungen werden in FloodRiskIIWerkzeuge(z. B. Lamellenprognose) weiterentwickelt und empfohlen (Habersack et al., 2009).

\subsubsection{Angepasste Nutzung durch die Raumplanung sicherstellen}

Sowohl rechtlich (Novelle des WRG in Bezug auf die Implementierung der EU Hochwasserrichtlinie) als auch technisch sollte die Erhaltung und Verbesserung der Überflutungsflächen vorrangig behandelt werden. Dazu soll eine gesetzliche Verankerung erfolgen. In FloodRisk II wurden Werkzeuge (z.B. die Floodplain Evaluation Matrix FEM, s h. Artikel drei in diesem Heft) entwickelt, die für die Erstellung eines Überflutungsflächenkatasters (soll u. a. den Summationseffekt in Kombination mit der WRG Novelle vermindern) herangezogen werden können. Die Einhaltung des minimalen flussmorphologischen Raumbedarfs sollte Eingang in die Raumplanung, Gefahrenzo- 
nenplanung etc. finden. Mit der Landwirtschaft besteht dringender Handlungsbedarf hinsichtlich Nutzung von Überflutungsflächen.

\subsubsection{Anreizsysteme zur Eigenvorsorge}

Aus den in FloodRisk II durchgeführten Projekten zum Objektschutz und mobilen Hochwasserschutz ergeben sich konkrete Vorschläge für die Umsetzung. Die Studien zum Objektschutz und mobilen Hochwasserschutz können als Grundlage für die Entwicklung von Normen und Standards in diesen Bereichen dienen.

\subsubsection{Hochwasserrelevante negative Entwicklungen erkennen}

In FloodRisk II wird eine verstärkte Einbeziehung des Feststoffhaushaltes und der Morphodynamik in Planungen als wesentlicher Teil des integrierten Hochwassermanagements vorgeschlagen. Dazu wurden konkrete Ansätze entwickelt (z. B. minimaler flussmorphologischer Raumbedarf) und auch der Querbezug zur Wasserrahmenrichtlinie und dem Nationalen Gewässerbewirtschaftungsplan (NGP) hergestellt (z.B. hinsichtlich Sedimentkontinuum). Eine Behandlung des Themas Sedimentkontinuum inklusive Feststoffhaushalt und Flussmorphologie im NGP ist aus Sicht von FloodRisk II unumgänglich.

\subsubsection{Planungen der öffentlichen Hand abstimmen}

Die begonnene Abstimmung aller Planungen der öffentlichen Hand sollte intensiv weitergeführt werden. Hilfreich ist in diesem Zusammenhang die neugegründete Stabstelle im Lebensministerium. Das umfasst die Abstimmung zwischen BWV, WLV, Wasserstraßenverwaltung, aber auch Raumplanung, Infrastrukturplanung und Katastrophenschutz.

\subsubsection{Schutzmaßnahmen, wo nötig}

Die gesetzliche Verankerung der Überflutungsflächen im WRG wird in FloodRisk II vorgeschlagen und die praktische Umsetzung in Hochwasserschutzprojekten sollte auf Basis eines Überflutungsflächenkatasters erfolgen. In Bezug auf die bestehenden Hochwasserschutzmaßnahmen gibt es konkrete Richtlinien zur Überwachung und Instandhaltung von z.B. Dämmen und Wildbachsperren. Weiters finden sich in FloodRisk II Aussagen zum Management der Vegetation (räumlich differenziertes Vegetationsmanagement) und Wildholz (integrales Wildholzmanagement).

\subsubsection{Notfallplanung und Katastrophen- schutzmaßnahmen ausbauen}

Das Bundesministerium für Inneres hat gemeinsam mit anderen Bundesministerien, den Bundesländern und den Dachorganisationen der großen Einsatzorganisationen eine längerfristige Strategie des staatlichen Krisen- und Katastrophenschutzmanagements erarbeitet.

Ein zentraler Aspekt - insbesondere für das Management von Naturkatastrophen - ist auch die flächendeckende Versorgung mit Einrichtungen des Katastrophenschutzes. Der Aufrechterhaltung des ehrenamtlichen Engagements im Katastrophenschutz kommt daher längerfristig eine wesentliche Bedeutung für den Erhalt der Qualität des gesamten Systems zu.

\subsubsection{Finanzielle Vorsorge sicherstellen}

Aus volkswirtschaftlicher Sicht gilt es vor allem, die vorhandenen Ressourcen bestmöglich einzusetzen. Die Bereitstellung einer soliden Informationsbasis über die Gefährdung vor Ort ist die dringlichste Aufgabe.

\subsubsection{Vorwarnung verbessern}

In FloodRisk II wird die weitergehende Umsetzung der Hochwasserprognosesysteme empfohlen, inklusive einer Evaluierung derselben, mit dem Ziel einer Optimierung und Verknüpfung mit neuen Instrumenten wie der Lamellenprognose.

\section{Schlussbemerkungen}

In Kombination mit der Implementierung der EU-Hochwasserrichtlinie besteht die einmalige Chance, die Vorschläge aus FloodRisk II direkt in gesetzlichen Vorgaben, Richtlinien und der Praxis umzusetzen. Klar ist, dass integriertes Hochwassermanagement nur im Zusammenwirken von Politik, Administration, Forschung UND der betroffenen Bevölkerung im Sinne einer Beteiligung am Risikodialog und im Rahmen der geforderten Partizipation erfolgreich ist. Wichtig ist die rasche Umsetzung der Empfehlungen aus FloodRisk II in die Praxis, denn es können und werden auch weiterhin Ereignisse auftreten, wie das Hochwasser 2009 unter Beweis gestellt hat.

\section{Danksagung}

Die Autoren bedanken sich beim Lenkungskreis, dem Kernteam, den WorkpackageleiterInnen, den TeilprojektleiterInnen und den vielen ProjektmitarbeiterInnen.

\section{Korrespondenz:}

Ao. Univ.Prof. DI Dr. Helmut Habersack Institut für Wasserwirtschaft, Hydrologie und Konstruktiven Wasserbau (IWHW), Department für Wasser-AtmosphäreUmwelt (WAU), Universität für Bodenkultur Wien, Muthgasse 107, A-1190 Wien, helmut.habersack@boku.ac.at MR DI Dr. Heinz Stiefelmeyer Bundesministerium für Land- und Forstwirtschaft, Umwelt und Bundesministerium für Land- und Forstwirtschaft, Umwelt und
Wasserwirtschaft, Abteilung VII 5, Marxergasse 2, 1030 Wien, heinz.stiefelmeyer@lebensministerium.at Ass.-Prof. Dr. Arthur Kanonier Department für Raumentwicklung, Infrastruktur- und Umweltplanung, Fachbereich für Rechtswissenschaften, TU Wien, Argentinierstraße 8, A-1040 Wien, Kanonier@law.tuwien. ac.a

Mag. Dr. Jochen Bürgel, MAS Umweltbundesamt GmbH., Abteilung Umweltfolgenabschätzung und Klimawandel, Spittelauer Lände 5 , 1090 Wien, jochen.buerge|@umweltbundesamt.at 